\title{
ANTIRADICAL AND ANTIHYPERGLYCEMIC ACTIVITY OF RASPBERRY POMACE
}

\section{(C) Tumbas Vesna ${ }^{1}$, Djilas Sonja ${ }^{1}$, Čanadanović-Brunet Jasna ${ }^{1}$, Ćetković Gordana ${ }^{1}$, Moreno Diego A. ${ }^{2}$, Vulić Jelena ${ }^{1}$, Savatović Slađana ${ }^{1}$}

${ }^{1}$ Faculty of Technology, University of Novi Sad, Bulevar cara Lazara 1, 21000 Novi Sad, Serbia ${ }^{2}$ CEBAS-CSIC, Food Science and Technology Department, P. O. Box 164, E-30100 Espinardo, Murcia, Spain

Plant foods and products are rich sources of a variety of biologically active compounds possessing wide range of biological activities such as hypolipidemic, antiplatelet, antitumor, antioxidant, and immunostimulating properties. Enzyme inhibitors have potential value, in many areas of disease control and treatment. $\alpha$-Glucosidase is a therapeutic target for type II diabetes, and $\alpha$-glucosidase inhibitors have been used in the clinic as alternative treatments for this disease. There is a growing interest to reduce waste and to study the possibility of using by-products, such as pomace from fruit processing industry, for isolation of bioactive compounds. For this purpose, raspberry pomace, Meeker variety, was extracted and screened for the presence of biologically active compounds with antiradical and $\alpha$-glucosidase enzyme-inhibitory activity. In order to characterize the raspberry pomace as a source of bioactive compounds, total polyphenol $(6.15 \mathrm{mg} / \mathrm{g}$ pomace), flavonoid $(3.87 \mathrm{mg} / \mathrm{g}$ pomace) and anthocyanin ( $0.66 \mathrm{mg} / \mathrm{g}$ pomace) contents in the extract were determined spectrophotometrically, while the presence of vitamin C $(1.41 \mathrm{mg} / \mathrm{g}$ pomace $)$ was determined by HPLC. Raspberry pomace extract exhibited antihyperglycemic activity, inhibiting $\alpha$-glucosidase activity at the concentration range from 0.04 to $0.63 \mathrm{mg} / \mathrm{ml}$. Inhibitory activity reached $50 \%$ applying $0.2 \mathrm{mg} / \mathrm{ml}$ of raspberry pomace extract. Antiradical activity of raspberry pomace extract, evaluated by ABTS assay, was $1.17 \mathrm{mM}$ Trolox equivalents at the concentration of $3.33 \mathrm{mg} / \mathrm{ml}$. Results obtained in this study show that rasberry pomace is a natural source of effective and safe bioactive compounds for development of functional foods to treat diabetes and oxidative stress related diseases.

\section{DOWNREGULATION OF CYP26B1 RNA BY WILLOW BARK, ITS ETOH-FRACTION AND BY THE ANTIDEPRESSANT IMIPRAMINE}

\section{(C) Koptina A. ${ }^{1,6}$, Kelber O. ${ }^{3}$, Zeitler H. ${ }^{2,}$ Abdel-Aziz H. ${ }^{3}$, Ludwig M. ${ }^{4}$, Wagner H. ${ }^{5}$, Ulrich-Merzenich G. ${ }^{1}$}

${ }^{1}$ Medical Clinic III, University of Bonn, Willhelmstr. 35-37, 53111 Bonn

${ }^{2}$ Medical Clinic I, University of Bonn, Sigmund-Freud-Str. 25, 53127 Bonn, Germany

${ }^{3}$ Steigerwald Arzneimittel GmbH, Havelstr. 5, 64295 Darmstadt, Germany

${ }^{4}$ Department of Clinical Chemistry and Clinical Pharmacology, University of Bonn, Sigmund-Freud-Str. 25, 53127 Bonn,

Germany

${ }^{5}$ Department of Pharmacy, LMU Munich, Butenandtstr. 5-13, 81377 Munich Germany

${ }^{6}$ Mari State Technical University, Yoshkar-Ola, Russia

CYP26B1 is a member of the cytochrome P450 26 family which catabolizes retinoids. Recently it was shown that CYP26B1 mRNA was increased in murine atherosclerosis and expressed in macrophagerich areas of human atherosclerotic lesions. A role of the CYP26B1 enzyme activity on atherosclerosis development by altering the availability of retinoid ligands was suggested and investigations on pharmacological CYP26PB1 inhibitors in the treatment of atherosclerosis and its complications proposed (1). The Netherlands study of depression and anxiety (NESDA) demonstrated a higher likelihood of subclinical atherosclerosis in persons with current depressive or anxiety disorders compared to healthy controls (2) substantiating a low grade inflammation in depression further. We exam- ined whether the anti-inflammatory agent willow bark, its salicin rich ethanol fraction and the tricyclic antidepressant imipramine target the gene-expression of CYPB26B1 and retinoid ligands in peripheral blood of rats in the Porsolt-Swimming Test (FST). Male Sprague Dawley rats $(n=12$ per group) were treated for 14 days p. o. with the willow bark extract STW 33-I (group A), its salicin rich fraction (group B), imipramine (group C). All three groups showed a reduction of the immobility time in the Porsolt swimming test, in comparison to an untreated control group as described earlier (3). Gene expression in peripheral rat blood of groups $A$, $B, C$ ( $n=4$ per group) were analysed by Agilent whole genome microarray, reverse transcriptase-PCR and compared to those of untreated controls $(n=4)$ (3). 